\title{
Haptic Human-Human Interaction Through a Compliant Connection Does Not Improve Motor Learning in a Force Field
}

\author{
Niek Beckers ${ }^{1}$, Arvid Keemink ${ }^{1}$, Edwin van Asseldonk ${ }^{1}$, and Herman van der \\ Kooij $^{1}$ \\ University of Twente, The Netherlands \\ n.w.m.beckers@utwente.nl
}

\begin{abstract}
Humans have a natural ability to haptically interact with other humans, for instance during physically assisting a child to learn how to ride a bicycle. A recent study has shown that haptic humanhuman interaction can improve individual motor performance and motor learning rate while learning to track a continuously moving target with a visuomotor rotation. In this work we investigated whether these benefits of haptic interaction on motor learning generalize to a task in which the interacting partners track a target while they learn novel dynamics, represented by a force field. Pairs performed the tracking task and were intermittently connected to each other through a virtual spring. Motor learning was assessed by comparing each partner's individual performance during trials in which they were not connected to the performance of participants who learned the task alone. We found that haptic interaction through a compliant spring does not lead to improved individual motor performance or an increase in motor learning rate. Performance during interaction was significantly better than when the partners were not interacting, even when connected to a worse partner.
\end{abstract}

Keywords: human-human interaction, motor learning, haptics, humanrobot interaction

\section{Introduction}

Haptic interaction between two humans comes naturally, for example while physically supporting a child which is learning to walk, carrying heavy items together or assisting a patient during physical rehabilitation. Interaction between humans concerning, for instance, joint action [13] and observational learning [1, 9, 7] have been studied in the past. Social interaction through gaming has shown improvement in motor learning, for instance because it makes the task more fun and motivating $[3,8]$. Haptic interaction - interaction through exerting forces onto each other - has only recently gained academic interest $[10,6,12,15,16,2,11]$.

A majority of the studies reported that haptic interaction increases the observed motor task performance. The seminal work by Reed and Peshkin [10] showed that haptically connected pairs were faster at acquiring a target in a 
ballistic reaching task compared to both subjects doing the same task alone. In another influencial paper, Ganesh et al. [6] showed that both continuously haptically interacting partners were better at a continuous tracking task than people practicing the task alone. Interestingly, they found that participants improved due to interaction, irrespective of the skill level of the partner they were interacting with. Even with a worse partner, tracking performance improved due to the haptic interaction.

Furthermore, results from Ganesh et al. [6] suggest that interaction improves individual motor learning of tracking a target while the cursor was visually rotated around a midpoint (a visuomotor rotation) on two aspects. First, interacting partners showed absolute better individual performance after learning to perform the tracking task compared to participants practicing the task alone. Second, the initial individual motor learning rate of interacting partners was faster. Results of another study, in which participants performed an unstable balancing task, suggested that haptic interaction does not increase motor learning rate and improves individual performance only under certain conditions [2]. However, to our knowledge, no further studies have been conducted to specifically investigate the benefits of interaction on motor learning or whether these benefits generalize to other motor learning tasks, for instance whether interaction benefits learning to perform a tracking task in a novel dynamic environment.

In this work we study the effect of haptic interaction on motor learning of a velocity-dependent force field specifically. Participants performed a collaborative continuous movement task in which they intermittently interacted through a virtual spring with a partner. We elicit motor learning by introducing novel dynamics - a force field [14] - to investigate whether improvements in motor learning due to haptic interaction in a visuomotor rotation task, as reported by [6], also generalize to other learning tasks. By comparing the individual motor performance of the interacting partners with a group that practiced the task alone, we infer whether haptic interaction indeed leads to improved motor learning. Based on the results of Ganesh et al. [6], we expected that haptic interaction also ameliorates motor learning in a tracking task in which the participants have to learn novel dynamics.

Contrary to previous studies, we found that haptic interaction neither leads to individual motor performance improvement, nor that motor learning rate is increased. We did find that motor performance during haptic interaction is significantly better than participants practicing the task alone. Furthermore, results show that interaction improves tracking performance even when connected to a worse partner - similar as in $[6,16]$. However, if a partner is around $40 \%$ worse in performance, no improvement is gained during interaction.

\section{Materials \& Methods}

\subsection{Robotic setup}

Participants held and moved their own identical robotic manipulandum, as is shown in Fig. 1. The robotic setup allowed arm movements in a 2D plane over 
A

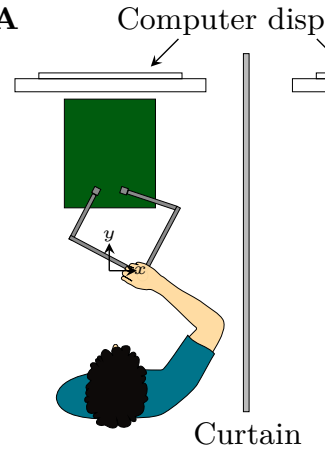

B

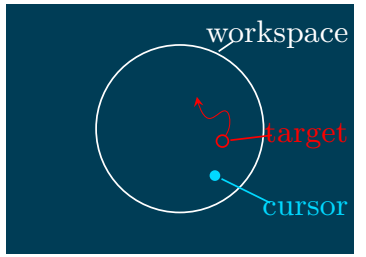

Fig. 1. Robotic setup and display. A Each participant had their own individual robotic handle and display. Each robot had the same relative coordinate system. A curtain obstructed the view of the other partner. B Each participant saw the same target and only their own cursor position on their own display.

a workspace that was constricted to a circle with a radius of $10 \mathrm{~cm}$. The robotic setups were admittance-controlled to emulate a virtual mass of $0.3 \mathrm{~kg}$ and a damping of $0.25 \mathrm{~N} \mathrm{~s} \mathrm{~m}^{-1}$ (isotropic over the workspace). Both robots were controlled with the real-time EtherCAT system using the TwinCAT3 master software which executed a compiled MATLAB Simulink model running at $1 \mathrm{kHz}$.

Each partner had their own display that showed the allowed workspace, target and their own cursor (see Fig. 1). Cursor and target movement were scaled to match the real-world movement of the robotic manipulandum.

\subsection{Task}

Forty participants (aged 19-35 years, 28 males and 12 females) performed the experiment in pairs, similar as in [6]. All participants performed the same planar tracking task. The goal was to track a target as accurately as possible on a display. The cursor was controlled by moving a robotic manipulandum. Each participant had his/her own robotic manipulandum and display; a curtain obstructed view of the other partner and the partner's display.

Tracking signal The participants tracked the continuously moving target during trials of $23 \mathrm{~s}$, including a lead-in time of $3 \mathrm{~s}$. The target movement was always the same for both partners in a pair. The target movement (in $\mathrm{cm}$ ) was defined as a sum-of-sines:

$$
\begin{aligned}
x(t)=2.87 \sin ( & 0.94 t-7.77)+2.71 \sin (1.26 t-8.53) \\
& +2.35 \sin (1.89 t-4.36)+1.80 \sin (2.83 t-3.79) \\
y(t)=2.71 \sin ( & 1.26 t-0.71)+2.53 \sin (1.57 t-3.45) \\
+ & 2.16 \sin (2.20 t+3.92)+1.64 \sin (3.14 t+4.93) .
\end{aligned}
$$


The tracking signal required hand movements over a circular workspace with a diameter of $20 \mathrm{~cm}$, a minimum velocity of $2.2 \mathrm{~cm} \mathrm{~s}^{-1}$, an average velocity of 7.9 $\mathrm{cm} \mathrm{s}^{-1}$ and a maximum velocity of $13.9 \mathrm{~cm} \mathrm{~s}^{-1}$. To prevent fast learning or other cognitive strategies, we chose a uniformally random start time for the signals $\left(t \in\left[t_{0}, t_{0}+20\right] \mathrm{s}, t_{0} \sim \mathcal{U}(0,20) \mathrm{s}\right)$ and we rotated the tracking signal randomly from a set of six predefined rotations: $\left(\varphi=\left[0, \frac{1}{3} \pi, \frac{2}{3} \pi, \pi, \frac{4}{3} \pi, \frac{5}{3} \pi\right] \mathrm{rad}\right)$.

Experiment design Each pair performed four blocks of 21 trials. Between blocks participants had a four-minute break. In blocks 2 and 3 a velocitydependent force field was introduced, with a planar force given by

$$
\left[\begin{array}{l}
F_{v, x} \\
F_{v, y}
\end{array}\right]=\left[\begin{array}{cc}
0 & -15 \\
15 & 0
\end{array}\right]\left[\begin{array}{l}
v_{x} \\
v_{y}
\end{array}\right]
$$

The force field allowed analysis of motor learning during the tracking task (see $[5,17])$ and, during haptic interaction between partners in a pair, the effect of interaction on motor learning. Pilot tests of our experiment design and previous work [5] confirmed that a force field in a continuous tracking task results in motor learning. The concept of a force field was explained to the participants; they were instructed to try to track the target as accurately as possible despite the forces. Furthermore, we asked participants not to stop tracking to learn the force field.

After each trial, the visual feedback was switched off and the robotic manipulanda were moved back to the start position by the robotic device, followed by a $20 \mathrm{~s}$ break.

Participants gave informed consent to participation in the study. The study was designed following the principles of the Declaration of Helsinki. An expedited assessment of the study by the Medical Ethical Review Board of the University of Twente (METC Twente) showed that the study poses minimal risk and under Dutch law does not need full medical ethical review.

Solo and interaction groups Twenty pairs were randomly assigned to a solo group or an interaction group (resulting in ten pairs per group). Pairs were not informed about which group they were in.

Connected and single trials Two types of trials were used in the experiment: connected and single trials. The interaction group interacted in alternating trials with each other through a haptic elastic force; these trials are denoted as connected trials. The elastic force allowed the partners to haptically interact, while being able to independently execute the tracking task.

The computer-generated elastic force consisted of a spring stiffness of $K_{s}=$ $150 \mathrm{~N} \mathrm{~m}^{-1}$ and damping $D_{s}=2 \mathrm{~N} \mathrm{~s} \mathrm{~m}^{-1}$

$$
\mathbf{F}_{s}=K_{s}\left(\mathbf{p}_{p}-\mathbf{p}_{y}\right)+D_{s}\left(\mathbf{v}_{p}-\mathbf{v}_{y}\right),
$$


where $\mathbf{F}_{s}$ is the elastic force exerted on a participant's hand, $\mathbf{p}_{p}$ and $\mathbf{v}_{p}$ and $\mathbf{p}_{y}$ and $\mathbf{v}_{y}$ are the partner's and the participant's own position and velocity, respectively. The elastic force was exerted onto both partners' hands by the robotic setup. If a partner moved away from the other partner, they both experienced a force pulling them toward each other (similar to [6]). The stiffness of the spring was chosen to be similar to the stiffness used in previous work [6]. Participants could feel the interaction force, yet the compliance was such that independent and active task execution was required; participants could not completely relax and let the force pull their hand along. The partners were not connected during the remaining trials; these trials are denoted as single trials.

The sequence of alternating single (S) and connected (C) trials (hence SCSCSCSCSCSCSCSCSCSCS) for the interaction group was the same for each block. Participants were not explicitly made aware whether the trial was a single or connected trial.

The solo group performed all trials alone (only single trials). For consistency, participants in the solo group always performed the task in pairs, but were never connected.

\subsection{Data analysis}

Motor performance was measured as the root-mean-square (RMS) of the tracking error, denoted by $E$ (in $\mathrm{cm}$ ), recorded at $1 \mathrm{kHz}$. Performance was calculated per partner for each trial. The performance during single trials represents the individual motor performance and is denoted by $E_{\mathbf{s}}$. For the interacting pairs during connected trials, $E_{\mathrm{c}}$ represents a participant's motor performance while haptically interacting with their partner.

To evaluate how haptic interaction influences each partner's individual motor learing, we plotted the performance of the single trials, denoted by $E_{\mathbf{s}}$, of the interaction group and compared them to the single performance of the same trials of the solo group. An exponential function

$$
E_{\mathbf{s}}=E_{A} e^{-\lambda t}+E_{\infty}
$$

was fitted to the learning curves of the single trials for each participant to estimate the learning rate $(\lambda)$. We also fit this function to the performances during connected trials of the interaction group. Goodness of fit was assessed using the $r^{2}$ value (note that we use $r^{2}$ to avoid confusion with the relative performance $R$, explained later on).

Similar to Ganesh et al. [6] and Takagi et al. [16], we examine the relation between improvement due to the haptic interaction and the relative performance difference between partners. This allows us to study whether interaction with a better or worse partner results in differences in benefits of the interaction for different skill levels. The improvement in performance due to interaction $(I)$ is calculated as

$$
I=1-\frac{E_{\mathrm{c}}}{E_{\mathrm{s}}}
$$


where $E_{\mathrm{c}}$ is the performance in a connected trial and $E_{\mathrm{s}}$ is the performance in the single trial following the connected trial. The relative performance $(R)$ of the partner you interact with is calculated by

$$
R=1-\frac{E_{\mathbf{s}, p}}{E_{\mathbf{s}}}
$$

where $E_{\mathbf{s}, p}$ is the partner's performance during the single trial and $E_{\mathbf{s}}$ is the participant's own performance during the same single trial.

To test which relative performance $R$ still yields improvement during interaction $I$, we fit the linear model $I=\beta_{0}+\beta_{1} R+\beta_{2} R^{2}$ to the data using a robust least-squares algorithm with bisquare weighting. The $95 \%$ confidence bounds of the model were also calculated.

Interaction forces due to the connection spring and total measured force applied directly to the robotic handle were recorded during each trial. The RMS of the interaction force $F_{I}$ indicates how much force participants exchanged during interaction trials. We also analyse how much RMS measured force $F_{M}$ was needed to complete the tracking task in the force field for comparison. Exponential models in the form of $F_{M, I}=F_{A} e^{-\omega t}+F_{\infty}$ were fit to the interaction force and measured force for the connected and single trials to quantify the change in force during learning and interaction. To study how the measured force increased or decreased during a connected trial compared to a single trial and how this varies with relative partner performance, we calculated the force ratio as the ratio of the measured force during a connected trial $F_{M, \mathrm{c}}$ and the force during the immediately following single trial $F_{M, \mathbf{s}}$ in the force field blocks per participant.

Since data were not normally distributed (assessed with the Lilliefors tests) we used non-parametric methods to test for significant effects. We used the Wilcoxon signed-rank tests for within-group tests (for instance to compare single and connected performance). The Mann-Whitney U test was used to test differences between independent samples (between the solo and interaction groups). The level of significance was set to $\alpha=0.01$.

\section{Results}

To investigate whether haptic interaction leads to improved individual motor learning, we compared motor performance of a tracking task between two groups: an interaction group and a solo group. Participants performed the tracking task in pairs. The partners in the interaction group were intermittently connected through a virtual spring through which they haptically interacted; these trials are denoted as connected trials. The partners were not connected in the remaining trials and served as catch trials (denoted as single trials) to assess the individual performance of each partner. The pairs in the solo group were never connected and thus only performed single trials. A force field was introduced to elicit motor learning. The performance of the interacting partners during their single trials is compared to the single performance of the solo partners. 


\subsection{Haptic Interaction Does Not Improve Individual Motor Learning}
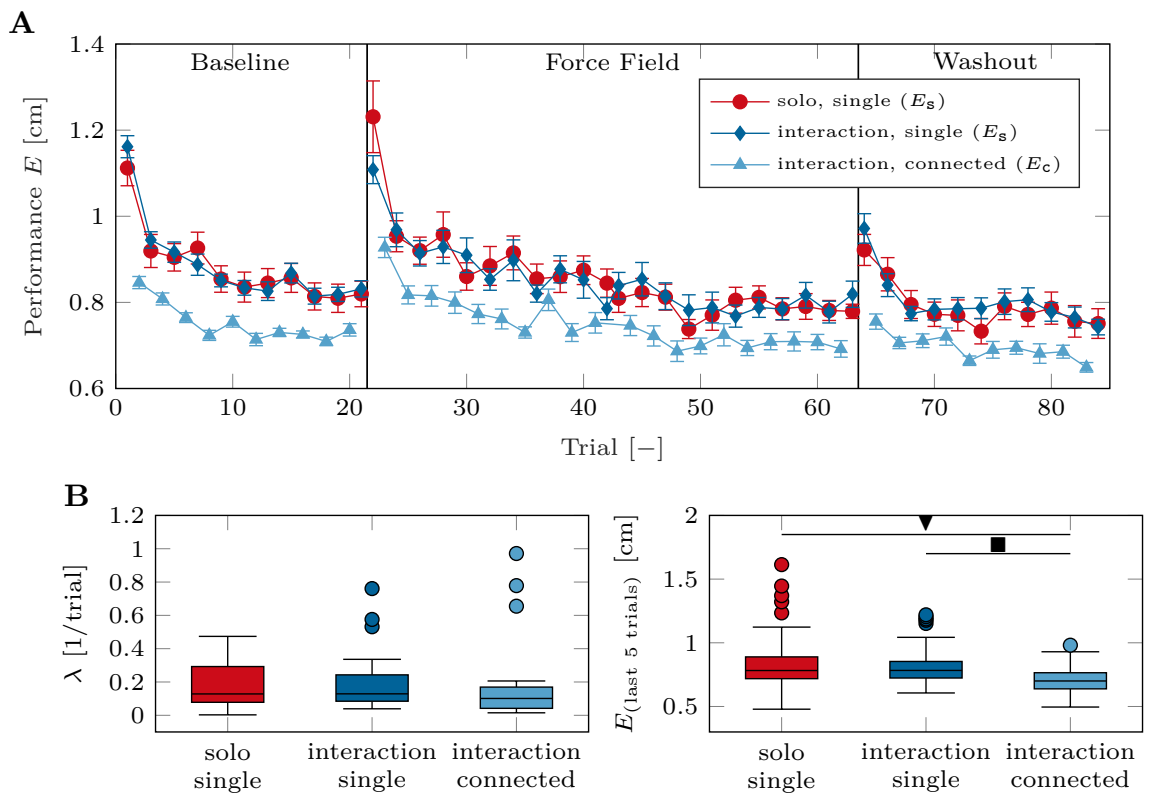

Fig. 2. Interaction though a compliant connection during learning a force field does not lead to improved individual motor learning. A Motor learning for the individual performances of all partners (interaction and solo groups) during the single trials $\left(E_{\mathbf{s}}\right)$, for all blocks. All errorbars indicate mean and standard error. B Individual learning rates $\lambda$, extracted from the fitted exponential learning curves of the force field blocks (all $r^{2}>0.6$ ). C Individual performance after learning the force field across groups. The box plots show the combined performance for all groups of the last five trials of the force field blocks. $\mathbf{~}$ : Wilcoxon signed-rank test, $W=4924, p<0.00001$. $\mathbf{v}$ : Mann-Whitney U test, $U=7431, p<0.00001$.

The motor learning curves (mean and standard error) of the individual motor performance during single trials for the interaction and solo groups for all blocks are shown in Fig. 2. We did not find any significant differences between the individual learning curves of the interaction and solo groups. Motor learning rates $\lambda$ of the learning curves during the force field blocks (Fig. 2B), extracted from Eq. 4, were not significantly different between the single trials of the interaction and solo groups. Fig. 2C shows the performance after learning based on the last five trials of the force field blocks, i.e. after learning the force field. We found no significant difference in individual performance after learning between the interaction and solo group. Hence, our results suggest that interaction through 
an compliant connection does not lead to improved individual performance or increased learning rate.

Individual motor performance improvement in the force field blocks was significant for all participants except four of the forty participants. For each participant, we compared the performance in the last eight single trials to the initial single trial in the force field using a sign test at $\alpha=0.05$. Furthermore, the absolute improvement for the solo and interaction group was in the same order of magnitude as [5], a study which also performed tracking tasks in a force field (note that they used an assistive/resistive force field).

Haptic interaction does lead to significant absolute improvement in motor performance during interaction (see the performance during the connected trials in Fig. 2A and Fig. 2C), corroborating the results of Ganesh et al. [6]. Performance in the last five connected trials was significantly better (lower performance score) compared to their own single trials and the single trials of the solo group (see Fig. 2C). As with the single trial performances, we did not see any differences in learning rate of motor performance during the interaction trials (Fig. 2B) compared to the single trials of the interaction group and solo group.

\subsection{Haptic Interaction Leads to Mutual Performance Benefits}

Since these results only partially corroborate the results of $[6,16]$, we wondered whether interaction leads to mutual benefits in improvement during interaction, even when connected to a worse partner; a key finding of [6]. Fig. 3A shows the relative improvement due to interaction $I$ versus relative partner performance $R$ (as introduced by [6]). Indeed, interacting with a better partner during a connected trial improves performance compared to the performance during a subsequent single trial. Interaction with a worse partner also improves performance, albeit less than interaction with a better partner. Our data show that the interaction benefits with a progressively worse partner are not limitless; interacting with a partner who performs $41.5 \%$ worse than your own performance leads to no significant improvement. The limit in relative performance $R$ at which no significant improvement $I$ was present is the point where the lower confidence bound of the fitted linear model $\left(I=\beta_{0}+\beta_{0} R+\beta_{2} R^{2}\right)$ intersects the abscissa $(I=0$, see Fig. 3A). Hence interaction leads to mutual benefits as in [6], but shows less benefit for interacting with progressively worse partners.

Interaction forces (median $=0.47 \mathrm{~N}$, last five connected trials) were almost three times lower than the measured force. The measured force during the last five connected trials (median $=1.49 \mathrm{~N}$ ) of the interaction group was slightly higher compared to their last five single trials (median $=1.45 \mathrm{~N}$ ), but not significant $(U=2826, p=0.067)$. We found no significant difference in measured force between the last five single trials of the interaction group and solo group $(W=2204, p=0.270)$. Interaction forces were almost threefold lower than the measured force (median $=0.47 \mathrm{~N}$, last five connected trials). The measured force $\left(F_{M}\right)$ during connected and single trials and interaction force $\left(F_{I}\right)$ all decreased significantly during the force field blocks (all 95\% model parameter confidence 
A

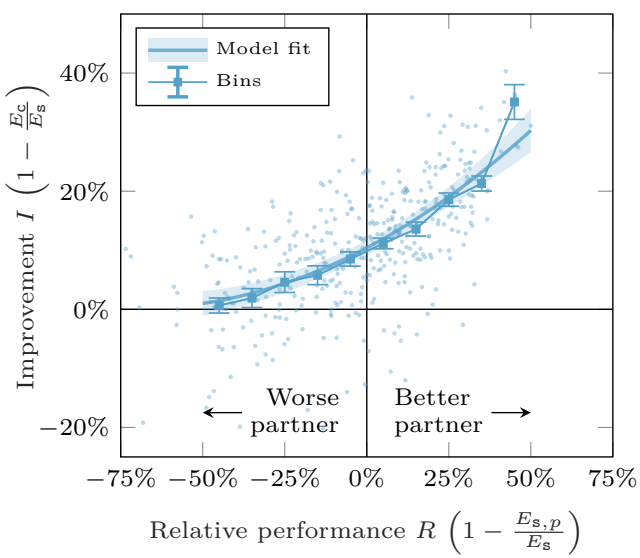

B

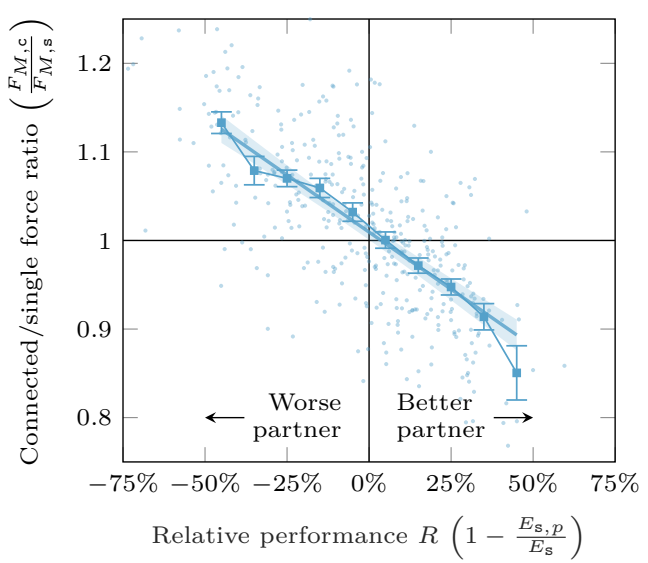

Fig. 3. Interaction yields mutual motor improvements. A The horizontal axis shows the relative task performance in percent of the partners in a pair. A relative performance of $-20 \%$ means that the partner's performance is $20 \%$ worse than the participant's own performance in a single trial. The vertical axis shows the relative improvement in performance during interaction compared to the single trial immediately following the interaction (connected) trial. B Ratio of the measured force during a connected trial and single trial with respect to relative partner performance. When you interact with a progressively worse partner, you increasingly apply more force compared to a single trial. The opposite happens when interacting with a better partner: the measured force decreases with a better partner.

intervals of the fitted exponential functions did not include zero). On initial exposure to force field, measured force during connected and single trials of the interaction group was slightly higher than the last five connected or single trials (difference of $0.058-0.169 \mathrm{~N}$ ). Interaction force was $0.074-0.123 \mathrm{~N}$ higher compared to the last five trials. Furthermore, as shown in Fig. 3B, during connected trials, participants that interacted with a better partner produced less force, whereas better partners produced more force relative to their subsequent single trial.

\section{Discussion}

In this work we investigated whether human-human haptic interaction with a partner through a virtual spring would result in improved individual motor learning compared to learning the task alone. We found that haptic interaction does not lead to improved individual motor performance or an increase in motor learning rate. Performance of the interacting partners during interaction was significantly better than when the partners were not interacting.

The main conclusion of this work - human-human haptic interaction does not improve individual motor learning - is in contrast with the findings of Ganesh et 
al. [6]. Although our study was methodologically similar to theirs, some aspects in the experiment could be the cause for the (partially) different results.

The type of learning task, force field or visuomotor rotation, could be an important factor in why we did not see improvement in individual motor learning. We chose for a force field for the following reasons. First, we wondered whether the effects on motor learning as observed by Ganesh et al. also generalized to tasks in which the haptic interaction forces are in the same domain as the forces needed to complete the task (e.g. counteracting the force field). We are not the first to do this: a recent study showed that human-human haptic interaction can be studied in a task in which participants performed a balancing task with unstable dynamics [2]. Furthermore, pilot tests and previous work [5] indicated that force fields can be used for motor learning assessment and yield significant motor learning, albeit with improvements in the order of millimeters, which is also confirmed by our results. A learning paradigm such as a visuomotor rotation could elicit more prominent learning. Second, haptic assistance when learning novel task dynamics, for instance during learning how to balance yourself on a bicycle, has in our opinion a higher out-of-the-lab validity compared to a visual perturbation [18]. It is possible that the benefits of human-human interaction are dependent on task characteristics, such as the learning paradigm. To investigate this hypotheses further, we are conducting a follow-up study using a visuomotor rotation as the learning paradigm.

Perhaps the motor task did not leave enough room for learning, hence the task did not facilitate the interacting partners to improve beyond their baseline performance. We performed (unpublished) tests in which interacting and solo partners performed the same tracking task but with more difficult tracking signals (signals with higher accelerations and velocities). The observed performances were worse (i.e. higher mean error $E$ per trial), however we did not see a significantly better motor improvement of the interaction group. Increasing the difficulty of a tracking task by increasing the difficulty of the tracking signal does not necessarily lead to 'better' motor learning curves. For instance, between-subject variability and non-linearity in tracking behavior (e.g. focus lapses, predictive tracking) are observed when increasing the tracking signal difficulty [4], complicating analysis.

Lastly, our data support the observation of [6] that haptic interaction leads to mutual performance improvement benefits: interaction improves performance for both partners, even for the partner interacting with a worse partner. Through computational modelling, Takagi et al. [16] posit that the mutual benefits during interaction exist because partners infer each other's movements through the haptic connection. In this study, however, one could argue that the forces due to moving through the force field 'mask' the interaction forces, making it difficult to distinguish between interaction forces and force-field-induced forces and hence infer your partner's movement. Still, interaction in a force field learning task leads to mutual improvement. Perhaps humans are indeed able to infer their partner's movement despite the ambiguitiy of the origin of the experienced force. However, our results may also provide support for alternative explanations for the mutual 
benefits during interaction, for instance that interaction implicitly counteracts irregular or erratic tracking behavior of both partners.

We also found that interacting with progressively worse partner reduces the improvement during interaction. When a partner is $41.5 \%$ worse in individual relative performance, interacting with that partner does not yield improvements for the participant. Our data do no go beyond a relative performance of $-50 \%$, so we do not know whether the trend observed in Fig. 3A continues to negative improvement or converges to zero improvement for progressively worse partners.

The interaction force $F_{I}$ due to the spring was approximately three times smaller than the measured force $F_{M}$ during a single or connected trial. Sawers et al. [11] showed that movement goals are communicated by small forces in a cooperative task, which might concur with the interaction force results we found. However, since the interaction forces are much smaller than the measured force and the force field and interaction forces regularly aligned in direction, it is questionable whether participants were able to reliably distinguish forces due to interaction or forces due to moving through a force field. The interaction forces may have been used to stabilize tracking behavior. This is corroborated by the presented force ratio data; it seems that better partners 'take over' or counteract the irregular or erratic tracking behavior of the worse partner, at the expense of a higher measured force during interaction. A more detailed analysis of the force profiles is necessary; although useful, lumping a force profile in a single parameter (RMS) results in loss of important features that could unveil aspects of interaction.

While our study provides additional results for assessing the efficacy of human-human interaction on motor learning, many key aspects were not taken into account in this study. Although we used a different learning paradigm than described in [6], we still do not know how task-dependent the effects of humanhuman interaction are on motor learning. In addition, we only tested one connection stiffness, a factor that plays an important role in physical interaction and subsequently learning. We intend to measure learning during interaction with different (in particular higher) connection stiffnesses. Our own pilot tests did show differences in improvement during interaction, where the worse partner increased more with higher connection stiffnesses, but no differences in learning. Furthermore, our analysis does not go into more detail on the differences in (absolute) motor skill of the interacting partners, and how the difference in motor skill influence the partners on an individual level. Currently, our analysis is performed on group level, whereas further investigation on within-participant effects is warranted.

Although we did not find direct evidence that human-human haptic interaction through a compliant connection leads to improved individual motor learning, the paradigm has many uses for assistive human-robot interaction or human-human interaction through robotics for learning applications, such as gaming and rehabilitation. Further studies are needed to identify the principles underlying human-human haptic interaction, such that human-robot interactions algorithms can haptically interact with a human in a more intuitive way. 


\section{References}

1. Aglioti, S.M., Cesari, P., Romani, M., Urgesi, C.: Action anticipation and motor resonance in elite basketball players. Nature Neuroscience 11(9), 1109-1116 (2008)

2. Avila Mireles, E.J., Zenzeri, J., Squeri, V., Morasso, P., De Santis, D.: Skill learning and skill transfer mediated by cooperative haptic interaction. IEEE Transactions on Neural Systems and Rehabilitation Engineering (2017)

3. Baur, K., Wolf, P., Riener, R., Duarte, J.E.: Making neurorehabilitation fun: Multiplayer training via damping forces balancing differences in skill levels. In: 2017 International Conference on Rehabilitation Robotics (ICORR, pp. 876-881. IEEE (2017)

4. Beerens, G.C., Damveld, H.J., Mulder, M., van Paassen, M.M., Van Der Vaart, J.C.: Investigation into Crossover Regression in Compensatory Manual Tracking Tasks. Journal of Guidance, Control, and Dynamics 32(5), 1429-1445 (2009)

5. Conditt, M.A., Gandolfo, F.: The motor system does not learn the dynamics of the arm by rote memorization of past experience. Journal of Neurophysiology (1997)

6. Ganesh, G., Takagi, A., Osu, R., Yoshioka, T., Kawato, M.: Two is better than one: Physical interactions improve motor performance in humans. Scientific Reports 4 (2014)

7. Granados, C., Wulf, G.: Enhancing motor learning through dyad practice: Contributions of observation and dialogue. Research Quarterly for Exercise and Sport 78(3), 197-203 (2007)

8. Mace, M., Kinany, N., Rinne, P., Rayner, A., Bentley, P., Burdet, E.: Balancing the playing field: Collaborative gaming for physical training. Journal of NeuroEngineering and Rehabilitation 14(1), 1 (2017)

9. Mattar, A.G., Gribble, P.L.: Motor Learning by Observing. Neuron 46(1), 153-160 (2005)

10. Reed, K.B., Peshkin, M.A.: Physical Collaboration of Human-Human and HumanRobot Teams. IEEE Transactions on Haptics 1(2), 108-120 (2008)

11. Sawers, A., Bhattacharjee, T., McKay, J.L., Hackney, M.E., Kemp, C.C., Ting, L.H.: Small forces that differ with prior motor experience can communicate movement goals during human-human physical interaction. Journal of NeuroEngineering and Rehabilitation 14(1), 8 (2017)

12. Sawers, A., Ting, L.H.: Perspectives on human-human sensorimotor interactions for the design of rehabilitation robots. Journal of NeuroEngineering and Rehabilitation 11(1) (2014)

13. Sebanz, N., Bekkering, H., Knoblich, G.: Joint action: bodies and minds moving together. Trends in Cognitive Sciences 10(2), 70-76 (2006)

14. Shadmehr, R., Mussa-Ivaldi, F.A.: Adaptive representation of dynamics during learning of a motor task 14(5), 3208-3224 (1994)

15. Takagi, A., Beckers, N., Burdet, E.: Motion Plan Changes Predictably in Dyadic Reaching. PLoS ONE 11(12), e0167,314 (2016)

16. Takagi, A., Ganesh, G., Yoshioka, T., Kawato, M., Burdet, E.: Physically interacting individuals estimate the partner's goal to enhance their movements. Nature Human Behaviour 1(3), 0054 (2017)

17. Tong, C., Flanagan, J.R.: Task-specific internal models for kinematic transformations. Journal of Neurophysiology 90(2), 578-585 (2003)

18. Wulf, G., Shea, C.H.: Principles derived from the study of simple skills do not generalize to complex skill learning. Psychonomic Bulletin \& Review 9(2), 185$211(2002)$ 